\section{El síndrome metabólico y la sensibilidad a la insulina en adolescentes obesos hispanos}

El síndrome metabólico genera un riesgo adicional de diabetes tipo 2 y de enfermedad cardiovascular en adultos, según los resultados del Tercer Informe del Panel de Expertos sobre la Detección, la Evaluación y el Tratamiento de la Hipercolesterolemia en Adultos (Third Report of the Expert Panel on Detection, Evaluation, and Treatment of High Blood Cholesterol in Adults, Adult Treatment Panel III). Recientemente se informó de que la prevalencia del síndrome metabólico ajustada según la edad en los adultos que participaron en la Tercera Encuesta Nacional de Salud y Nutrición (NHANES III 19881994), llevada a cabo en los Estados Unidos de América, era de $23,7 \%$ y especialmente alta en las personas de origen hispano $(31,9 \%)$, lo cual parece asociarse con la alta prevalencia de obesidad en ese grupo de población. La elevada prevalencia de obesidad entre escolares hispanos residentes en los Estados Unidos se conjuga con el aumento en la incidencia de diabetes tipo 2 y de intolerancia a la glucosa. Esto podría deberse a una mayor resistencia a la insulina que, a su vez, podría estar asociada con alteraciones metabólicas.

Los objetivos de este estudio fueron: determinar la prevalencia del síndrome metabólico y sus componentes individuales entre adolescentes hispanos obesos; establecer el papel que desempeña la sensibilidad a la insulina en el síndrome metabólico (independientemente de la composición corporal); y explorar la asociación entre la sensibilidad a la insulina y los componentes individuales del síndrome metabólico en este grupo de población.

Los autores estudiaron a 126 adolescentes de 8 a 13 años de edad de origen hispano (73 del sexo masculino y 53 del sexo femenino) con un índice de masa corporal $(\mathrm{IMC}) \geq$ percentil 85 y que no eran diabéticos, pero que tenían antecedentes familiares de diabetes tipo 2 .

A los adolescentes que participaron en la investigación se les realizó la prueba oral de tolerancia a la glucosa y la prueba de sobrecarga intravenosa de glucosa modificada por la insulina con muestreos frecuentes (insulin-modified frequently sampled iv glucose tolerance test). Además, se les tomaron las medidas antropométricas (talla, peso y circunferencia de la cintura) y la tensión arterial. La composición corporal se determinó mediante absorciometría de rayos $X$ de energía doble. El diagnóstico de síndrome metabólico se realizó cuando un adolescente presentaba al menos tres de las alteraciones siguientes: obesidad abdominal (circunferencia de la cintura $\geq$ percentil 90 para su edad, sexo y grupo étnico); triglicéridos $\geq$ percentil 90 para su edad y sexo; colesterol por lipoproteínas de alta densidad $(\mathrm{HDL}) \leq$ percentil 10 para su edad y sexo; hipertensión arterial (tensión arterial sistólica o diastólica > percentil 90, ajustada según su talla, edad y sexo); e intolerancia a la glucosa.

La prevalencia de los componentes individuales del síndrome metabólico se calculó como el porcentaje de adolescentes con obesidad abdominal, bajo colesterol HDL, hipertrigliceridemia, hipertensión arterial e intolerancia a la glucosa. Los participantes en el estudio fueron clasificados en cuatro grupos según el número de componentes del síndrome metabólico que presentaran (ninguno, uno, dos, o tres componentes o más).

Aproximadamente $90 \%$ de los participantes en el estudio presentaban al menos un componente del síndrome metabólico: $22 \%$ tenían uno, $38 \%$ presentaban dos y $30 \%$ tenían tres componentes o más. Alrededor de $10 \%$ de los adolescentes estudiados presentaban cuatro o cinco componentes del síndrome metabólico. En relación con el sexo, solo se encontró una diferencia significativa en la prevalencia de hipertrigliceridemia (9\% en las adolescentes frente a $15 \%$ en los varones; $P<0,001)$. En comparación con los adolescentes que solo presentaban riesgo de obesidad (IMC $\geq$ percentil $85 \mathrm{y}<$ percentil 95), entre los obesos (IMC $\geq$ percentil 95) se encontraron más casos de obesidad abdominal ( $0 \%$ frente a $76 \%$, respectivamente), de colesterol HDL bajo ( $48 \%$ frente a $71 \%$, respectivamente) y de tensión arterial sistólica elevada ( $0 \%$ frente a $27 \%$, respectivamente) $(P<0,05)$. Por último, $6 \%$ del total de adolescentes estudiados presentaban valores elevados de colesterol total plasmático y $10 \%$ tenían valores elevados de colesterol por lipoproteínas de baja densidad (LDL).

Según mediciones directas, la sensibilidad a la insulina en esta cohorte estuvo asociada directamente con las concentraciones de colesterol HDL e inversamente con las concentraciones de triglicéridos y con la tensión arterial, pero no con la glucosa al cabo de 2 horas, y estas relaciones eran independientes de la grasa corporal. La sensibilidad a la insulina disminuyó a medida que disminuía el número de compo- 
nentes del síndrome metabólico. Estos resultados apuntan a que la resistencia a la insulina es un componente central del síndrome metabólico en los adolescentes hispanos obesos que tienen antecedentes familiares de diabetes tipo 2 y que esta resistencia a la insulina está asociada con un perfil metabólico desfavorable. Estos resultados confirman los hallazgos de otros estudios realizados en adolescentes estadounidenses, según los cuales una alta proporción de adolescentes obesos puede tener un mayor riesgo de padecer diabetes tipo 2 y de enfermedad cardiovascular.

Estos resultados indican que puede ser de suma importancia mejorar la resistencia a la insulina para prevenir tanto la diabetes tipo 2 como la enfermedad cardiovascular prematura en este grupo de riesgo compuesto por adolescentes estadounidenses de origen hispano. (Cruz Ml, Weigensberg MJ, Huang TTK, Ball G, Shaibi GQ, Goran MI. The metabolic syndrome in overweight Hispanic youth and the role of insulin sensitivity. J Clin Endocrinol Metab 89:108-113, 2004.)

\section{Efectos de la malaria placentaria sobre la transmisión del VIH de madre a hijo en Rikai, Uganda}

La malaria y la infección por el virus de la inmunodeficiencia humana tipo 1 (VIH-1) se encuentran entre las enfermedades infecciosas más frecuentes en África subsahariana y la coinfección es habitual en áreas donde la malaria es endémica. La frecuencia y la gravedad de la malaria son mayores en mujeres embarazadas que en mujeres no embarazadas y conduce a desenlaces adversos en el embarazo. De manera similar, existe una mayor tasa de nacimientos con bajo peso, de partos prematuros y de mortalidad infantil entre los niños nacidos de madres positivas al VIH en comparación con los nacidos de madres seronegativas. Por lo tanto, la coinfección de la malaria y el VIH-1 durante el embarazo puede tener consecuencias graves para los recién nacidos, por lo que la prevención de estas infecciones durante el embarazo constituye una prioridad de la salud pública.

En este estudio se evaluó el efecto de la malaria placentaria sobre la transmisión del VIH de madre a hijo, a fin de mejorar el conocimiento actual sobre este complejo problema.

Entre 1994 y 1999 se realizó un ensayo aleatorizado comunitario de control de enfermedades de transmisión sexual (ETS) en Rakai, Uganda, con el fin de prevenir la infección por VIH. Como parte de este estudio se evaluaron los efectos del tratamiento para ETS en la salud de la madre y del niño durante el embarazo. Las mujeres embarazadas que dieron su consentimiento fueron asignadas a un grupo de tratamiento con antibióticos o a un grupo de control que recibió la atención habitual en caso de ETS. Las mujeres participantes fueron entrevistadas y se les tomaron muestras para el diagnóstico de VIH y de ETS. Se hizo un seguimiento de las madres y sus hijos para conocer el peso de los recién nacidos y la edad gestacional en el momento del parto, así como para establecer si hubo transmisión de la ETS de la madre al hijo. La presencia del VIH en los recién nacidos se determinó a la edad de 4-6 semanas mediante la reacción en cadena de la polimerasa. Además, se realizaron análisis histopatológicos de las placentas.

De las 668 placentas procedentes de mujeres cuyo estatus con relación a la infección por VIH era conocido, $62(9,3 \%)$ presentaban parásitos de malaria. De las 155 madres positivas al VIH, 21 (13,6\%) presentaban señales de infección parasitaria en la placenta, en comparación con 8,0\% (41/510) de las madres seronegativas $(P=0,039)$. La tasa de transmisión del VIH de madre a hijo fue de $40 \%(6 / 15)$ en los casos de malaria placentaria y de 15,4\% (12/78) cuando no se observaron signos de infección parasitaria en la placenta $(P=0,027)$. Según el análisis de la varianza, la transmisión de madre a hijo estuvo significativamente asociada con la carga viral de la madre (riesgo relativo [RR] 2,32; intervalo de confianza de $95 \%$ [IC95\%] 1,66 a 3,24, por cada aumento de $1 \log _{10}$ de la carga viral) y con la presencia de parásitos en la placenta (RR 2,60; IC95\% 1,16 a 5,84). El hecho de ser el primer embarazo no estuvo asociado con la transmisión de la infección de la madre al hijo (RR 1,17; IC95\% 0,74 a 2,34). La tasa de riesgo ajustada de la transmisión de madre a hijo asociada con la malaria placentaria fue de RR 2,89 (IC95\% 1,12 a $7,52)$ después de hacer el ajuste según la carga de VIH (RR 2,85; IC95\% 1,53 a 5,32, para cada incremento de $1 \log _{10}$ de la carga viral).

La mediana de la carga viral de VIH en los recién nacidos fue mayor en los hijos de madres infectadas también con malaria que en los hijos de madres infectadas solo por el VIH (4,58 frente a 3,77 $\log _{10}$ copias $/ \mathrm{mL}$, respectivamente; $P=0,13$ ).

Estos resultados confirman que la prevalencia de la infección placentaria con malaria entre las madres positivas al VIH es mayor que entre las seronegativas y que la inmunodeficiencia resultante de la infección por el VIH podría estar afectando a la respuesta inmunitaria contra la malaria. La coinfección con malaria y VIH durante el embarazo estuvo asociada significativamente con la infección de madre a hijo y esta asociación fue más fuerte después de hacer el ajuste según la carga viral de VIH de la madre. La carga viral de VIH de los recién nacidos fue mayor en los niños infectados nacidos de madres con malaria placentaria, aunque esto no fue confirmado estadísticamente. 
El aumento del riesgo de transmisión de la infección de la madre al hijo asociada con la malaria placentaria puede explicarse mediante diferentes mecanismos. La inflamación provocada por la malaria puede dañar la placenta, lo que podría aumentar el intercambio sanguíneo entre la madre y el feto y facilitar la transmisión del VIH in utero. La malaria también ocasiona un aumento de la expresión del correceptor de la quemocina CCR5 en los macrófagos de la madre y en las células de Hofbauer del feto, lo que podría hacer al niño más susceptible a la infección por el VIH.

Estos hallazgos pueden tener importancia para la salud pública, ya que las intervenciones dirigidas a prevenir la malaria durante el embarazo podrían reducir la transmisión de la infección por el VIH de la madre al hijo y esto podría aumentar las posibilidades de que la profilaxis antirretroviral tenga eficacia. Se necesitan con urgencia estudios aleatorizados sobre el control intensivo de la malaria en mujeres positivas al VIH. (Heena Brahmbhatt; Godfrey Kigozi; Fred Wabwire-Mangen; David Serwadda; Nelson Sewankambo; Tom Lutalo; Maria J. Wawer; Carlos Abramowsky; David Sullivan; Ronald Gray. The effects of placental malaria on mother-to-child HIV transmission in Rakai, Uganda. AIDS 2003; 17(17): 2539-2541.)

\section{El gen de la lipoproteína-lipasa está asociado con la resistencia a la insulina en estadounidenses de origen mexicano}

El síndrome de resistencia a la insulina (también llamado síndrome metabólico) está formado por un conjunto de factores asociados con el aumento del riesgo de cardiopatía isquémica. Este síndrome afecta a más de $20 \%$ de los adultos en los Estados Unidos de América y las mayores prevalencias ajustadas por la edad corresponden a los estadounidenses de origen mexicano. La resistencia a la insulina - acompañada o no por otras manifestaciones del síndrome metabólico- está asociada con un aumento del riesgo de accidentes cardiovasculares e incluso de muerte. Por ello se ha puesto una atención especial en identificar los determinantes genéticos asociados con el síndrome de resistencia a la insulina.

Estudios recientes indican que la gran variabilidad genética de los humanos se describe mejor mediante grupos de polimorfismos asociados, $\mathrm{CO}^{-}$ nocidos como haplotipos. La identificación de un haplotipo asociado con el aumento o con la disminución del riesgo de padecer una enfermedad puede facilitar la identificación de la variante funcional que influye efectivamente en ese riesgo. Mediante este tipo de análisis se demostró reciente- mente la asociación de haplotipos del terminal carboxílico del gen de la lipoproteína-lipasa (LPL) con la arteriopatía coronaria. El objetivo de este trabajo fue explorar si existe alguna asociación entre las variaciones del gen de la LPL y la resistencia a la insulina utilizando esos haplotipos.

Los casos índice de esta investigación fueron estadounidenses adultos de origen mexicano con antecedentes de infarto del miocardio (documentado mediante un electrocardiograma o la historia clínica hospitalaria), con diagnóstico de aterosclerosis (mediante arteriografía coronaria), o con antecedentes de derivación aortocoronaria o angioplastia. En el estudio participaron los casos índice y sus cónyuges (generación parental), así como sus descendientes mayores de 18 años de edad y sus respectivos cónyuges (generación de descendientes). Se obtuvo ADN para el análisis genotípico de todos los participantes de ambos grupos; además, a los miembros de la generación de descendientes se les realizaron pruebas para caracterizar sus fenotipos metabólico y cardiovascular, entre ellas la prueba de resistencia a la insulina mediante pinzamiento euglucémico.

Se realizó el análisis genotípico de los marcadores del gen de la LPL de 485 participantes provenientes de 80 familias. Se estudió el fenotipo de 291 descendientes de 74 familias.

Con la estructura del árbol genealógico y los datos genotípicos de los individuos de una misma línea genealógica se construyeron los conjuntos más probables de haplotipos paternos y maternos de los marcadores estudiados para cada persona del árbol genealógico. Mediante este método se logró asignar haplotipos a 475 de las 485 personas genotipadas, entre ellas 284 de las 291 personas que además fueron fenotipadas (199 descendientes y 85 cónyuges de descendientes).

En promedio, las 291 personas (112 hombres y 179 mujeres) a quienes se les determinó la resistencia a la insulina presentaban obesidad, lo que podría explicar en parte el grado de resistencia a la insulina observado. No obstante, se conoce que los estadounidenses de origen mexicano tienen una predisposición a la adiposidad visceral, a la hiperinsulinemia y a la resistencia a la insulina.

Estos resultados indican que en esta población se pueden encontrar cuatro haplotipos del gen de la LPL que confieren distinta sensibilidad a la insulina. El haplotipo 1 fue el más frecuente de todos. Se observó que a medida que disminuía el número de cromosomas con el haplotipo 1, la sensibilidad a la insulina disminuía progresivamente. Por su parte, los portadores del haplotipo 4 fueron los menos sensibles a la insulina. Los datos disponibles indican que existe una variante funcional en los cromosomas del haplotipo 1 que favorece la sensibilidad a la insulina 
y una variante en los cromosomas que portan el haplotipo 4 que provoca la resistencia a la insulina. Hasta el momento no es posible distinguir si el locus nucleotídico responsable de este comportamiento es el mismo en ambos haplotipos o si estas variaciones están localizadas en diferentes sitios del gen.

Este es el primer estudio en el que se ha utilizado la sensibilidad a la insulina -medida mediante pinzamiento euglucémico- como indicador del fenotipo a fin de investigar su asociación con el gen de la LPL. El vínculo de este gen con la mayor o menor resistencia a la insulina quedó demostrado mediante la asociación estadística entre los haplotipos del gen de la LPL y el grado de resistencia a la insulina. La relación entre las variaciones del gen de la LPL y la sensibilidad a la insulina determinada mediante su medición fisiológica directa en la población estudiada ofrece una prueba convincente de esta asociación.

Teniendo en cuenta los resultados de estudios anteriores que demuestran la asociación de variantes individuales del gen de la LPL con la aterosclerosis, la dislipidemia, la obesidad y la hipertensión, se recomienda utilizar los haplotipos descritos aquí en investigaciones diseñadas para evaluar la asociación de este gen con los componentes del síndrome de resistencia a la insulina, especialmente en la población estadounidenses de origen mexicano. (Goodarzi MO, Guo X, Taylor KD, Quiñones MJ, Saad $\mathrm{MF}$, Yang $\mathrm{H}$, et al. Lipoprotein lipase is a gene for insulin resistance in Mexican Americans. Diabetes 2004;53:214-220.)

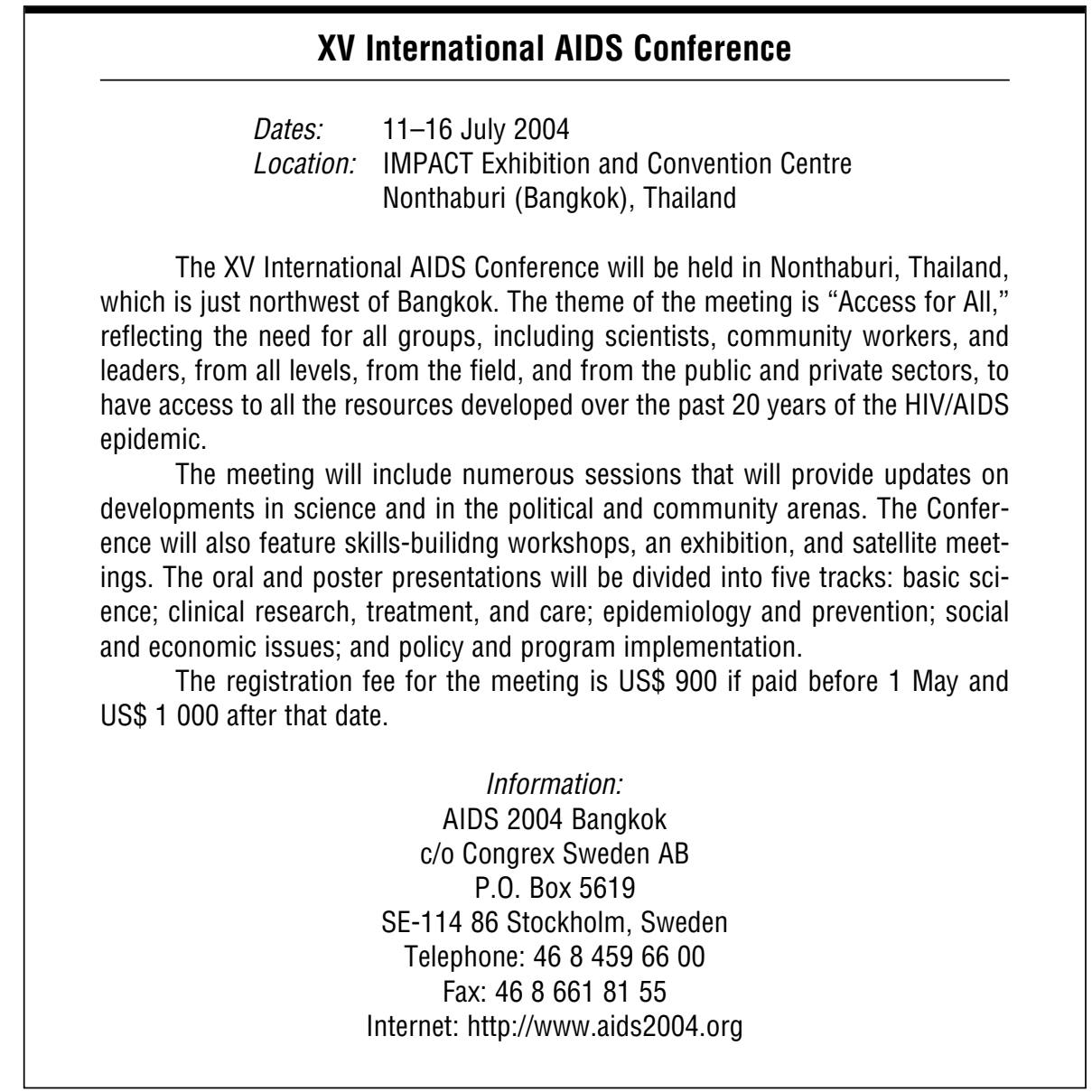

